\title{
Constraints on the subsurface structure and density of the nucleus of Comet 67P/Churyumov-Gerasimenko from Arecibo radar observations
}

\author{
P. Kamoun ${ }^{1}$, P. L. Lamy ${ }^{2}$, I. Toth ${ }^{2,3}$, and A. Herique ${ }^{4}$ \\ 1 Université de Nice Sophia Antipolis \& Thales Alenia Space, Cannes, France \\ e-mail: paul . kamoun@thalesaleniaspace.com \\ ${ }^{2}$ Laboratoire d'Astrophysique de Marseille, UMR 7326, CNRS \& Aix-Marseille Université, Marseille, France \\ 3 Konkoly Observatory, MTA CSFK CSI, 1121 Budapest, Hungary \\ ${ }^{4}$ UJF-Grenoble 1 \& CNRS-INSU, Institut de Planétologie et d'Astrophysique de Grenoble, UMR 5274, 38041 Grenoble, France
}

Received 30 January 2014 / Accepted 8 June 2014

\begin{abstract}
Context. Little is known about the internal structure of cometary nuclei. In addition to understanding their accretion in the early solar nebula and their subsequent evolution in the solar system, we find this question to be of acute and timely interest in the case of 67P/Churyumov-Gerasimenko (hereafter 67P/C-G) due to be visited by the Rosetta spacecraft in the second half of 2014. In particular, the successful landing of the Philae surface module depends critically upon the bulk density of the nucleus and the structure of its surface layer.

Aims. In addition to fostering our general knowledge of these properties, it is important to exploit all possible information to assist in preparing the delivery of Philae.

Methods. We performed an in-depth analysis of the observations done with the radar system of the Arecibo Observatory in November 1982 when comet 67P/C-G had a close encounter with Earth at a geocentric distance of 0.4 AU taking our present knowledge of the properties of its nucleus (size, rotational state) into account.

Results. In the absence of a detectable radar echo, we determined a maximum radar cross section of $0.7 \mathrm{~km}^{2}$, leading to a maximum radar albedo of 0.05 . This low albedo probably results from a combination of a low radar reflectivity material and a lightly packed upper layer of the nucleus with substantial roughness ( $r m s$ slope of $\approx 55^{\circ}$ ), consistent with its low thermal inertia. Based on radar observations of other cometary nuclei and asteroids, it is unlikely that the albedo can be lower than 0.04 so that we were able to constrain the dielectric permittivity of the subsurface layer to a narrow range of 1.9 to 2.1. Laboratory measurements and our modeling of mixtures of ice and dust have led to a porosity in the range of approximately 55 to $65 \%$ and a density in the range of $\approx 600$ to $\approx 1000 \mathrm{~kg} \mathrm{~m}^{-3}$ for the top $\approx 2.5 \mathrm{~m}$ layer of the nucleus. This would be the bulk density range for a homogeneous nucleus and would place the success of the landing at risk, but an inhomogeneous nucleus with an overall density below this range remains a possibility.
\end{abstract}

Key words. comets: general - comets: individual: 67P/Churyumov-Gerasimenko - techniques: radar astronomy

\section{Introduction}

Cometary nuclei are considered to be among the most primitive objects in the solar system, and their properties are thought to preserve a record of the conditions that existed in the early solar nebula. Of particular interest is their internal structure, which may give clues to the process of their accretion and their subsequent evolution in the solar system. In the case of 67P/Churyumov-Gerasimenko (hereafter 67P/C-G), the question is even more acute and timely because of the forthcoming landing of the Philae surface element of the Rosetta mission in late 2014. The safe landing of Philae remains a critical issue (see Fig. 1 of Hilchenbach et al. 2004; Lamy et al. 2008) depending upon the size (now well constrained) and the density (poorly constrained) of the nucleus.

Following the pioneering work of Kamoun et al. (1982a, $1982 \mathrm{~b}$ ), it has been realized that comets coming close enough to Earth could produce detectable radar echoes from their nucleus (and also from their large coma grains), allowing the derivation of constraints on their radar albedos and on their surface densities. The review article of Harmon et al. (2004) summarizes the properties inferred from six nuclei detected from ground-based observatories: radar albedos in the range 0.04-0.1 and nucleus surface densities in the range $500-1500 \mathrm{~kg} \mathrm{~m}^{-3}$. Comets detected thereafter (Harmon \& Nolan 2005; Harmon et al. 2006, 2011) follow the same pattern with the notable exception of the nucleus of 8P/Tuttle (Harmon et al. 2010) which has a radar albedo of 0.15 and a relatively well-compacted surface with a density in the range $900-1800 \mathrm{~kg} \mathrm{~m}^{-3}$.

The main motivation for using radar to study comets lies in the fact that, unlike other ground-based astronomical techniques, radar offers the possibility of obtaining information simultaneously on its shape, size, and rotational state, on its surface scattering properties, and on the structure and density of its subsurface down to a few meters. Radar observations of comet 67P/C-G were performed in November 1982 when it had a close encounter with Earth at a geocentric distance of $0.4 \mathrm{AU}$, leading to an upper limit of the radius of the nucleus of approximately $3 \mathrm{~km}$ (Kamoun 1983; Kamoun et al. 1999), actually not far from the presently accepted value of the effective radius of $2 \mathrm{~km}$ (Lamy et al. 2008). At that time, nothing was known about this nucleus so that the analysis could not proceed further. This is no longer the case because tremendous effort has been 
made to characterize the nucleus in preparation for the Rosetta mission and, particularly, for the landing of Philae (Lamy et al. 2007, 2008).

In this article, we reconsider the analysis of the 1982 radar observations of Kamoun et al. (1999) in the light of our present knowledge of the nucleus of $67 \mathrm{P} / \mathrm{C}-\mathrm{G}$. The observations are first recalled in Sect. 2. After reviewing our present knowledge of the nucleus of $67 \mathrm{P} / \mathrm{C}-\mathrm{G}$ in Sect. 3, we perform a complete analysis of the data in Sect. 4. We then present our results and their interpretation in Sect. 5. We finally discuss the question of the density of the nucleus in Sect. 6 and then conclude.

\section{Radar observations of comet $67 P / C-G$}

The observations of comet $67 \mathrm{P} / \mathrm{C}-\mathrm{G}$ were performed using the $2.38 \mathrm{GHz}(12.6 \mathrm{~cm}) \mathrm{S}$ band of the continuous wave $(\mathrm{CW})$ radar system of the Arecibo Observatory and consisted of eight daily sessions from 8 to 15 November 1982. Each session lasted less than two hours because of the limited sky coverage of the Arecibo antenna and was broken into cycles. A cycle consisted of the transmission of a circularly polarized nearly monochromatic wave with a transmitted power $P_{\mathrm{t}}$ of $400 \mathrm{~kW}$ during a time approximately equal to the expected round-trip time of the radar signal. It was followed by reception of the echo for nearly the same duration in the two (orthogonal) senses of circular polarization: opposite to that transmitted OC (for "opposite circular"), and the same as transmitted SC (for "same circular"). Ephemerides based on the orbit of the comet calculated from previous optical observations were used to point the telescope and to adjust the receiver frequency. Following the radar observations, a new ephemeris was calculated using all the astrometric data available from 1975 through November 1982. It appeared that the new ephemeris and the one used for the radar observations had an offset of approximately $0.8 \mathrm{~Hz}$ and local differences of less than $0.2 \mathrm{~Hz}$ implying no significant smearing of the echo during the observing period. Table 1 summarizes the observational circumstances and the system parameters.

The data reduction and analysis procedures have been described in detail by Kamoun (1983) and summarized in Kamoun et al. (1982b, see their Note No. 4). The primary parameter derived from the radar echo is the radar cross-section $\sigma$ of the target obtained by integrating the echo Doppler spectrum so as to retrieve the total received power $P_{\mathrm{r}}$. These two quantities are linked by the radar equation:

$P_{\mathrm{r}}=\frac{P_{\mathrm{t}} G^{2} \lambda^{2} \sigma}{(4 \pi)^{3} \Delta^{4}}$

where $G$ is the effective antenna gain, $\lambda$ the wavelength of the radar system, and $\Delta$ the geocentric distance of the comet. The analysis then considers the spectral distribution of the received echo in the different channels of the receiver for the two polarization states OC and SC. The bandwidth and shape of the spectrum are determined by the nucleus shape and orientation, as well as by the intrinsic angular scattering law of its surface. Table 2 displays the values of the signal-to-noise ratio in the nine spectral elements for the weighted sum of all the data. The width of the spectral elements is $1.1 \mathrm{~Hz}$ and frequency increases from right to left. The spectrum thus extends over $9.9 \mathrm{~Hz}$, which is ample enough to cover the estimated bandwidth of $7.7 \mathrm{~Hz}$. As noted above, the differences between the subsequently published ephemeris and the one used for the radar observations are much less than the $1.1 \mathrm{~Hz}$ channel spacing so that there was no significant smearing of the echo during the observing period and no need to consider additional channels in the analysis. The central one (number 186) corresponds to the a priori center of the echo. Polarization properties of the radar echo are useful for characterizing the surface of the nucleus. From the OC echo, one mostly learns about the quasi-specular scattering properties of the surface since a single reflection reverses the sense of circular polarization. From the SC echo, one learns of the depolarization by wavelength-scale roughness or multiple scattering properties. Whereas both OC and SC channels were available for $67 \mathrm{P} / \mathrm{C}-\mathrm{G}$, the signal-to-noise ratio of our measurements is not sufficient to investigate the polarization properties of the nucleus of $67 \mathrm{P} / \mathrm{C}$ $\mathrm{G}$. The powers received in the two senses of polarization are therefore combined to obtain the radar cross-section.

The analysis then proceeds by smoothing the weighted sum of the data for each single day of observation, as well as for the combination of the eight days, using optimal filters. These filters are defined by considering that the echo spectral shape can be fitted to that of a sphere using a standard scattering law of the form $\cos ^{n} \theta$ as a function of incidence angle $\theta$. A uniform scattering law corresponds to $n=1$ and a Lambert law to $n=2$. Then the echo spectrum can be simply expressed as $M\left\{1-\left[2\left(f-f_{0}\right) / B\right]^{2}\right\}^{n / 2}$, where $M$ is the maximum amplitude of the echo, $B$ is its total bandwidth, and $f_{0}$ represents the offset of the center of the spectrum from the prediction based on the ephemeris. We obtain a maximum signal-to-noise ratio of about three times the standard deviation for the combination of all days of observation using an estimated bandwidth of $7.7 \mathrm{~Hz}$ and $n=1$. In 1982, nothing was known on the nucleus of $67 \mathrm{P} / \mathrm{C}$ $\mathrm{G}$, and Kamoun et al. (1999) considered that this result was insufficient to claim a detection. Using very conservative estimates of the rotational period, of the orientation of the axis of rotation, and of the radar scattering efficiency, they obtained an upper limit of the radius of the nucleus of approximately $3 \mathrm{~km}$. Our knowledge of the nucleus of $67 \mathrm{P} / \mathrm{C}-\mathrm{G}$ has drastically improved in the meantime in preparation for the Rosetta mission, thus allowing a more refined analysis of the 1982 radar observations.

\section{Present knowledge of the nucleus of comet $67 \mathrm{P} / \mathrm{C}-\mathrm{G}$}

Lamy et al. (2007) presented a comprehensive review of the overall properties of the nucleus of comet $67 \mathrm{P} / \mathrm{C}-\mathrm{G}$. In particular, the shape and the rotational properties were derived by inverting a set of light curves coming from Hubble Space Telescope (Lamy et al. 2006) and ground-based (Lowry et al. 2006) observations. Lamy et al. (2008) later ascertained its size by combining these visible data with thermal measurements obtained with the Spitzer Space Telescope (SST). A more recent shape model has been proposed by Lowry et al. (2012), which benefits from additional, subsequent ground-based observations at large heliocentric distances, although these authors recognize that this condition (required to insure minimal coma contamination), seriously limit the viewing geometry and therefore, is a handicap for three-dimensional reconstruction. One main difference between the two models stems from the different inversion procedures implemented, in a broad stroke non-convex (Lamy et al. 2007) and convex (Lowry et al. 2012). However, they converge to a rather spherical nucleus with typical asphericity of $20 \%$ to $25 \%$. In both cases, the size comes from scaling the calculated thermal light curves to the SST data of Lamy et al. (2008). The model of Lowry et al. (2012) allows a better fit to the SST data and was therefore retained in the present study. Direct measurements of asteroids visited by spacecrafts have shown that inversion cannot 
P. Kamoun et al.: Internal structure of Comet 67P from Arecibo radar observations

Table 1. Observational circumstances and system parameters of the Arecibo radar observations of comet 67P/C-G.

\begin{tabular}{lcccccccccr}
\hline \hline \multicolumn{1}{c}{ Date } & $\mathrm{UT}$ & $r_{\mathrm{h}}$ & $\Delta$ & $\alpha$ & $\xi$ & $\mathrm{DB}$ & $\mathrm{RTT}$ & $G$ & $T$ & $t$ \\
1982 & $\mathrm{hr}$ min & $\mathrm{AU}$ & $\mathrm{AU}$ & $\mathrm{deg}$ & $\mathrm{deg}$ & $\mathrm{Hz}$ & $\mathrm{s}$ & $\mathrm{dB}$ & $\mathrm{K}$ & $\mathrm{s}$ \\
\hline November 8 & 0730 & 1.307 & 0.412 & 33.7 & 124.0 & 60,960 & 71.1 & 411.49 & 55 & 939 \\
November 9 & 0728 & 1.307 & 0.410 & 33.5 & 124.2 & 57,807 & 71.3 & 409.34 & 47 & 3130 \\
November 10 & 0727 & 1.306 & 0.408 & 33.2 & 124.5 & 54,628 & 71.3 & 407.30 & 50 & 3130 \\
November 11 & 0725 & 1.306 & 0.406 & 32.9 & 124.7 & 51,480 & 71.4 & 405.38 & 43 & 1305 \\
November 12 & 0724 & 1.306 & 0.404 & 32.6 & 125.0 & 48,303 & 71.1 & 403.58 & 51 & 2738 \\
November 13 & 0722 & 1.306 & 0.403 & 32.3 & 125.2 & 45,155 & 71.3 & 401.89 & 46 & 2809 \\
November 14 & 0721 & 1.306 & 0.401 & 32.0 & 125.5 & 41,978 & 71.2 & 400.31 & 51 & 3050 \\
November 15 & 0719 & 1.307 & 0.400 & 31.7 & 125.7 & 38,830 & 71.0 & 398.85 & 55 & 3314 \\
\hline
\end{tabular}

Notes. $r_{\mathrm{h}}, \Delta$ : heliocentric and geocentric distances. $\alpha, \xi$ : solar phase angle and aspect angle. DB: Doppler broadening of the echo. RTT: the round-trip time. $G, T$ : effective antenna gain and system temperature. $t$ : integration time.

Table 2. Unsmoothed spectra of the echo power for the two senses of polarization OC and SC.

\begin{tabular}{cccccccccc}
\hline \hline Spectral element \# & 182 & 183 & 184 & 185 & 186 & 187 & 188 & 189 & 190 \\
\hline Echo Power (OC) in $\sigma_{\text {rms }}$ & 0.7 & 1.1 & -0.9 & 0.8 & -0.1 & 0.6 & 2.6 & 1.6 & -0.1 \\
Echo Power (SC) in $\sigma_{\text {rms }}$ & -0.8 & -0.1 & -0.9 & 0.6 & 2.8 & -0.8 & 1.5 & 2.0 & -0.6 \\
\hline
\end{tabular}

produce accurate shapes but only gross properties, even when a large number of light curves at different geometries is available; therefore, the differences between the two models of the nucleus are most likely within their uncertainties.

The observations analyzed by Lowry et al. (2012) cover the longest time interval so far and have resulted in a refined determination of the sidereal rotational period $P=12.76 \mathrm{~h}$ and of the orientation of the rotational axis defined by its ecliptic coordinates $\lambda=78 \pm 10^{\circ}$ and $\beta=+58 \pm 10^{\circ}$.

The question we now face is whether significant changes occurred between the radar observations of $11 / 1982$ and the time period 3/2003-5/2007 used in the Lowry et al. 2012 analysis approximately corresponding to 3.5 orbital periods (of 6.57 years). First the surface erosion of the nucleus is negligible. Indeed, Lamy et al. (2007) estimated that $4 \%$ of the surface is on the average actively contributing to the water production, and they found that the active areas of $67 \mathrm{P} / \mathrm{C}-\mathrm{G}$ typically erode at a rate of approximately $5 \mathrm{~m}$ per apparition. The question of the evolution of the rotational state is more complex and has been investigated by Gutiérrez et al. (2005), see also a summary in Lamy et al. (2007). Taking all the available observational constraints into account (e.g., water production rate at perihelion, size, spin period), these authors performed numerical simulations for a large set of initial conditions, including several body shapes, various initial angular momenta (including the possibility of complex rotation), and different activity patterns. Depending upon these conditions, the rotational period may evolve from a fraction of an hour to approximately $1.5 \mathrm{~h}$ (in the worst case) per apparition. A reasonable estimate is $\approx 1$ h over three apparitions, or about $10 \%$, which will change the Doppler broadening proportionally. This is too small a change to affect the analysis in this paper.

For the evolution of the direction of the rotational axis, Gutiérrez et al. (2005) find that changes of up to about $10^{\circ}$ are possible after every orbit. However, because of the rather spherical shape of the nucleus of $67 \mathrm{P} / \mathrm{C}-\mathrm{G}$, this is not going to affect the apparent projected area $A_{\mathrm{p}}$ of the nucleus much, which was seen in November 1982 during the radar observations. Therefore, we adopt the present values as a conservative approach and display in Fig. 1 the temporal variation of this parameter and derive a

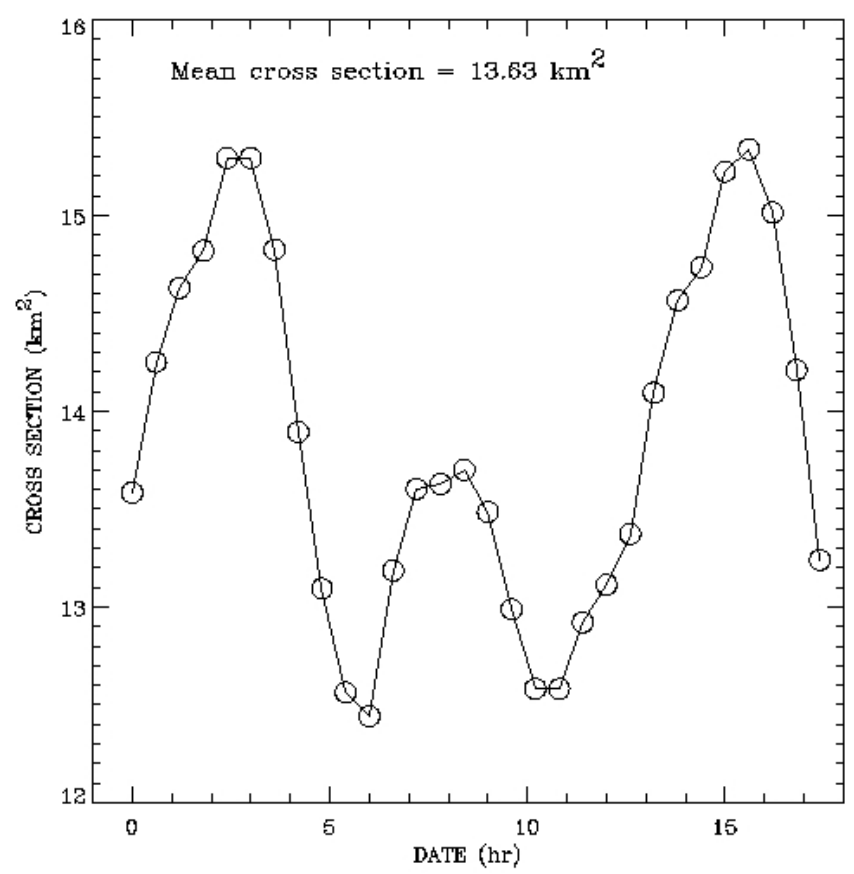

Fig. 1. Geometric cross-section of $67 \mathrm{P} / \mathrm{C}-\mathrm{G}$ as it rotates over 1.3 rotations using the shape and rotational state from Lowry et al. (2012) projected back to the time of the radar observations (8-15 November 1982).

mean value of $13.63 \mathrm{~km}^{2}$ (which corresponds to an effective radius of $2.08 \mathrm{~km}$ ). This is the value that we used in the analysis of the radar observations since the echo spectrum was averaged over all observations. The amplitude of the variation is only $\pm 10 \%$ with the nucleus spending very short times at the extrema; therefore, the mean value is an excellent estimate in the present case. The corresponding viewing angle $\xi$ ranged from $124^{\circ}$ to $125.7^{\circ}$ as given in Table 1 . 


\section{New analysis of the radar data}

The Doppler broadening of the echo (so-called "limb-to-limb" bandwidth) $B$ is related to the apparent projected area $A_{\mathrm{p}}$ through the equation:

$$
B=\frac{8 \sqrt{\pi A_{\mathrm{p}}} \sin \xi}{\lambda P_{\text {rot }}}
$$

where $P_{\text {rot }}$ is the rotational period of the nucleus. Using the above parameters, we find $B=7.1 \mathrm{~Hz}$, a result that could not be obtained in 1982 because the values of the parameters were not known. Given this value of $B$ and the knowledge of the standard deviation of the noise fluctuations per spectral element $\sigma_{\text {rms }}=0.1 \mathrm{~km}^{2}$, we can determine a more accurate estimate of the maximum radar cross section $\sigma_{\max }$ of the nucleus. We consider that, in the absence of a detectable echo, an upper limit of three standard deviations can be adopted for the maximum signal-to-noise ratio per spectral element.

The new value of $B$ is close to the estimated value of $7.7 \mathrm{~Hz}$ (a difference of $8 \%$ ) so that the optimum value $n=1$ for the scattering law as obtained in Sect. 2 is unaffected. Therefore, using $B=7.1 \mathrm{~Hz}$ and $n=1$, we calculate $\sigma_{\max }$ with an optimum combination of cells via the following equation (Kamoun et al. 1982b):

$\sigma_{\max }^{2}=\sum_{i=-m}^{m}\left[3\left(1-\left(2 f_{i} / B\right)^{2}\right)^{n / 2}\right]^{2} \sigma_{\mathrm{rms}}^{2}$

where $f_{i}=f-f_{0}$ for each considered spectral element, and $m$ the largest integer inferior or equal to $B / 2$, and we obtain $\sigma_{\max }=$ $0.7 \mathrm{~km}^{2}$.

\section{Interpretation}

Before interpreting the above results, let us point out that, contrary to optical and infrared observations that measure the light scattered or thermally emitted by a thin surface layer, radio waves penetrate much deeper below. Adopting a standard estimate of approximately ten wavelengths for packed soils, we find that the Arecibo radar beam penetrated to a depth of about $1.2 \mathrm{~m}$ and even more in the case of a porous medium.

\subsection{Albedo}

The geometric radar albedo is obtained using the standard equation:

$\hat{\sigma}=\sigma / A_{\mathrm{p}}$

which leads to a maximum value of 0.05 for the nucleus of 67P/C-G. The most reliable radar albedos determined so far are 0.05 for 2P/Encke (Harmon \& Nolan 2005), 0.15 for 8P/Tuttle (Harmon et al. 2010), 0.045-0.054 for 103P/Hartley 2 (using the EPOXI-based dimensions of the nucleus, Harmon et al. 2011), 0.08 for $26 \mathrm{P} /$ Grigg-Skjellerup, 0.04 for C/IRAS-Araki-Alcock (C/IAA), and 0.10 for C/Sugano-Saigusa-Fujikawa (C/SSF) (Harmon et al. 2004). However, adopting an effective radius of $3.5 \mathrm{~km}$ for C/IAA that Groussin et al. (2010) obtained from their in-depth analysis and interpretation of all visible, infrared, and radio observations raises its radar albedo to 0.06 . Consequently, with a maximum value of 0.05 , the nucleus of $67 \mathrm{P} / \mathrm{C}-\mathrm{G}$ belongs to a class of very low radar albedo objects represented by 2P/Encke and 103P/Hartley 2.
We enlarge our comparison to other minor bodies, although larger in size than the cometary nuclei. According to the radar surveys of 74 asteroids conducted by Magri et al. (2007) and Shepard et al. (2010), two Tholen class CP asteroids, 247 Eukrate (0.039) and 324 Bamberga, have radar albedo $\hat{\sigma}<0.05$, precisely 0.039 for the former and 0.041 for the latter ones. (1 Ceres also has a low albedo, namely 0.041 , but the comparison with nuclei is inappropriate.) We therefore conjecture that it is unlikely that the radar albedo of the nucleus of $67 \mathrm{P} / \mathrm{C}-\mathrm{G}$ can be lower than 0.04 . It is therefore constrained to the range $0.04-0.05$, the probable result of a combination of a low radar reflectivity material and a lightly packed upper layer of the nucleus, the latter being consistent with its low thermal inertia (Lamy et al. 2008; Lowry et al. 2012).

This result implies that the radar beam probed the nucleus at depths greater than the ten wavelengths noted in the introductory remark. Coincidentally, the optical albedo $(\approx 0.04$, Lamy et al. 2008) and the radar albedo have about the same values. This general trend was noticed by Harmon et al. (2004) but was considered not to be significant. Both albedos depend upon composition and roughness, but the optical albedo reflects the properties of a thin surface layer, whereas the radar albedo involves scattering taking place meters below the surface. The former is likely to be more sensitive to composition and the latter to bulk roughness.

\subsection{Surface and subsurface properties}

As explained in Sect. 2, when using a scattering law of the form $\cos ^{n} \theta$, the echo spectrum of comet $67 \mathrm{P} / \mathrm{C}-\mathrm{G}$ is best fit with $n=1$ corresponding to a uniform scattering law and implying substantial roughness, here again consistent with its low thermal inertia. As pointed out by Harmon et al. (2004), if the scattering is assumed predominantly specular, then the roughness can be estimated from geometric optics (Mitchell et al. 1995). In that case, the rms slope $\theta_{\mathrm{r}}$ of the surface roughness is related to $n$ by

$\theta_{\mathrm{r}}=\arctan (\sqrt{2 / n})$

yielding $\theta_{\mathrm{r}} \approx 55^{\circ}$ for $67 \mathrm{P} / \mathrm{C}-\mathrm{G}$. Since $\theta_{\mathrm{r}}$ is not very sensitive to $n$, all nuclei measured so far fall in the range $45-55^{\circ}$.

\subsection{Dielectric properties}

We follow the procedure of Harmon et al. (2004) to derive the dielectric permittivity using their equations reproduced below. Assuming again that the scattering is predominantly specular, the square of the Fresnel coefficient at normal incidence $\rho_{0}$ is related to the radar albedo $\hat{\sigma}$, and the backscattering gain $g$ via $\rho_{0} \approx \hat{\sigma} / g$. For a $\cos ^{n} \theta$ scattering law in the geometrics optics, approximation $g=(n+2) /(n+1)$, yielding $g=3 / 2$ in our case. The real part of the dielectric permittivity $\varepsilon$ is given by

$\varepsilon=\left(1+\rho_{0}^{1 / 2}\right)^{2} /\left(1-\rho_{0}^{1 / 2}\right)^{2}$

Our maximum value of the radar albedo (0.05) leads to a dielectric constant of the surface layer of 2.1 and a putative minimum albedo of 0.04 , to 1.9 . We therefore adopt a value $\varepsilon \approx 2.0$ as representative of the subsurface of the nucleus of $67 \mathrm{P} / \mathrm{C}-\mathrm{G}$.

The interpretation of this result can be first approached by separately considering the two main phases of cometary material, water-ice and refractory material.

Laboratory measurements of dry snow (defined as a mixture of ice particles and air voids and henceforth assimilated to 
porous ice) by Hallikainen et al. (1986) and their compilation of previous works, and later by Matzler (1996), show that the real part of its dielectric permittivity is independent of temperature and frequency in the microwave region and that it is a function of snow density alone. Figure 4 of Hallikainen et al. (1986) indicates a range of 1.2 to 3.2 as a function of density with a permittivity of 2.0 corresponding to $\approx 530 \mathrm{~kg} \mathrm{~m}^{-3}$, hence a porosity of $\approx 40 \%$.

Campbell \& Ulrichs (1969) measured the dielectric properties of a wide variety of rocks (36 samples) and mineral powders (25 samples) at two wavelengths, 0.45 and $35 \mathrm{GHz}$, in the framework of interpreting lunar radar observations. Since the lower wavelength is close to that of our observation and based on the fact that the values of the permittivity vary only slightly between the above two wavelengths, their results are adequate to our analysis. All measured rocks have permittivities exceeding 2.4 for a few of them (porous rocks, such as pumice and volcanic ash shale) and 5.0 for the bulk of them, thus ruling out solid rocks for the subsurface layer of the nucleus of $67 \mathrm{P} / \mathrm{C}-\mathrm{G}$. For powdered rocks, their measurements indicate that the permittivity is prominently a function of powder density obeying the Rayleigh formula with a weak dependence on temperature and no dependence on particle size distribution or compressive history of the powder. Calculating the permittivity of all 25 powders at a density of $1000 \mathrm{~kg} \mathrm{~m}^{-3}$ using the Rayleigh formula, they found that all results fall in the narrow range 1.9-2.1, precisely the range we determined for the nucleus of $67 \mathrm{P} / \mathrm{C}-\mathrm{G}$. In fact, they concluded that "a dielectric constant of 2.0 seems a reasonable estimate for most powdered rocks at this density". Such a density implies porosity in the range 65 to $70 \%$ based on the bulk densities of minerals expected to be present in cometary nuclei (see discussion in the next section). Another interesting result concerns the penetration depth: according to their Fig. 14, it exceeds 20 wavelengths at $0.45 \mathrm{GHz}$ for most of their samples (in particular olivine powders) at a density of $1000 \mathrm{~kg} \mathrm{~m}^{-3}$. This indicates that the Arecibo radar beam had probably penetrated the nucleus of $67 \mathrm{P} / \mathrm{C}-\mathrm{G}$ to a depth on the order of $2.5 \mathrm{~m}$.

This first approach strongly imposes that the subsurface of this nucleus is quite porous. Whereas the case of pure porous ice is excluded, the second case of a thick $\approx 2.5 \mathrm{~m}$ thick layer of regolith is conceivable but unlikely because ice is expected to be closer to the surface. For instance, the Deep Impact (DI) observations of $9 \mathrm{P} /$ Tempel 1 suggest that a surface layer of thickness less than $1 \mathrm{~m}$ is depleted in ice (A'Hearn 2008). From their model of the dust mantle formation of comet $67 \mathrm{P} / \mathrm{C}-\mathrm{G}$, Rosenberg \& Prialnik (2009) find that its thickness varies over its surface from $1 \mathrm{~cm}$ to about $10 \mathrm{~cm}$, much less than the expected penetration depth of the radar beam. Therefore, in a second approach, we investigate the dielectric permittivity of various samples of porous mixtures of ice and dust.

Laboratory measurement of porous material are challenging for technical reasons and are not available for porosity greater than $\approx 50 \%$ (Heggy et al. 2012); in fact, those of Campbell \& Ulrichs (1969) were limited to $40 \%$. An alternative approach consists in expressing the permittivity of a mixture from that of its (pure material) phases using mixing formulas. Different formulas have been established based on theoretical or empirical approaches, assuming some known geometry of the inclusions and neglecting the interactions between phases, see the review of Sihvola (2004). We first consider the classical Maxwell-Garnet mixing law, which assumes spherical inclusions in a bulk material. For a three-phase mixture (dust, water ice, and vacuum in our case), the M-G formulation can be inverted, thus providing the fraction of two phases as a function of the fraction

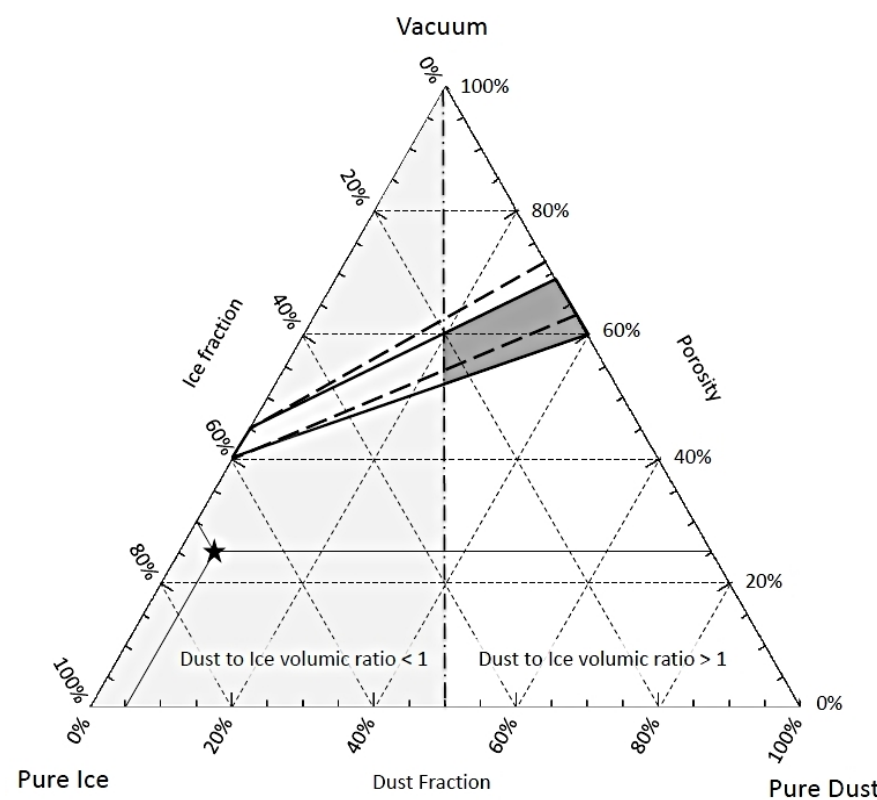

Fig. 2. Ternary diagram dust/ice/porosity volumetric fractions for cometary material. To help read this diagram, an example is displayed (star symbol) corresponding to $25 \%$ porosity, $5 \%$ dust, and $70 \%$ ice. See text for details of the different lines and shaded regions.

of the third one. We next introduce the more general Looyenga mixing law as an alternative and consider all compositions of the surface material bracketed by the above two laws to limit model sensitivity. The ice permittivity is taken equal to 3.2 corresponding to hexagonal water ice, the only form of ice that can be present at or close to the surface for thermodynamical reasons. A first dust permittivity of $\approx 5.8$ is obtained from the laboratory measurements of two meteoritic samples: LEW 85320 L5-type-meteorite and MET 01260 LL5-type-meteorite (Heggy et al. 2012). A second, somewhat larger permittivity of 7.0 corresponds to the equivalent permittivity of a mixture of $40 \%$ serpentine, $41,5 \%$ montmorillonite, $5.8 \%$ olivine, $8.8 \%$ magnetite, and $3.9 \%$ pyrrhotite, representative of the mineralogical composition of the Orgueil C-type meteorite and calculated by Beck \& Buttarazzi (priv. comm.). These two values of 5.8 and 7.0 are taken as mean values for the whole surface/subsurface averaging possible thermal effects (expected to be modest based on the laboratory measurements reported above).

The results are presented in a ternary diagram (Fig. 2) where the three axes corresponds to the volumetric fractions of dust, ice, and vacuum. The two continuous lines bound a first region where the calculated permittivity is equal to 2 as derived from the radar observations, using a dust permittivity of 5.8: the upper line corresponds to the Maxwell-Garnet law and the lower one to the Looyenga law. The two broken lines bound a second region with the same conditions as above except that it corresponds to a dust permittivity of 7.0. We can see that this parameter has very little influence $(<5 \%)$ in the present case of high porosities, much less than the mixing laws. The star in the figure indicates how to read the ternary diagram.

We now introduce an additional limitation on the ternary diagram coming from a constraint on the dust-to-ice mass ratio based on our present understanding of the composition of cometary nuclei. Traditionally and particularly in most thermophysical models, a ratio of 1 is assumed (e.g., De Sanctis et al. 2006 for a model of the nucleus of $67 \mathrm{P} / \mathrm{C}-\mathrm{G}$ ), although there is little direct evidence for this value. Observations of cometary 
comae are of no real help as the coma composition changes with heliocentric distance not to mention the problem of detecting the largest dust grains, which requires infrared, millimetric, or even radar measurements. Based on observations with IRAS, Sykes $\&$ Walker (1992) found that trail comets including 67P/C-G lose the bulk of their mass in the large refractory trail particles and are found to have a median refractory/volatile mass ratio of $\approx 3$. They suggested that comets in general may be more like "frozen mudballs" than the canonical "dirty snowballs". Recent millimetric observations of comet 103P/Hartley 2 by Boissier et al. (2014) indicate a dust-to-gas ratio in the range 2 to 6 . The analysis of the excavated material by the impact on the nucleus of comet 9P/Tempel 1 to a depth of about $15 \mathrm{~m}$ offers an independent estimate. From spectral observations of the ejecta performed with SST, Lisse et al. (2006) found a dust-to-gas mass ratio of at least 1.3 in the SST beam. Whereas the total mass of excavated ice is well constrained to $\approx 4.5 \times 10^{6} \mathrm{~kg}$ from $\mathrm{OH}$ remote observations (Küppers et al. 2005), this is less so for the dust as the ejecta was only remotely observed in the visible. Jorda et al. (2007) estimate the total mass of released dust to 1$14 \times 10^{6} \mathrm{~kg}$ truncated at a size of $100 \mu \mathrm{m}$, which they considered could be largely underestimated. Based on their own estimates, Küppers et al. (2005) conclude that the dust-to-ice ratio is probably considerably higher than 1 , suggesting that comets are "icy dirtballs" rather than the canonical "dirty snowballs" in line with the conclusion of Sykes \& Walker (1992). We adopt a conservative lower limit range of 2.5 to 3.5 because this further simplifies the derivation of the constraint on the dust-to-ice volumetric ratio to be introduced in the ternary diagram. Indeed, as discussed in the next section, this is also our preferred range for the density of the dust phase so that we can simply impose the condition that the dust-to-ice volumetric ratio is less than $\approx 1$. This eliminates half of the ternary diagram (light gray region) bounded by the vertical dashed-dotted line.

The allowed region (where the calculated permittivity is equal to 2) consequently shrinks to the dark gray quadrilateral in the case of a dust permittivity equal to 5.8. The corresponding quadrilateral for a dust permittivity equal to 7.0 is not highlighted but can be easily visualized as bounded by the two dashed lines. Varying the dust-to-gas mass ratio would cause the vertical dashed-dotted line to rotate around the top summit of the ternary diagram but in a very restricted angular range unless extreme values are considered. Therefore the allowed region is not very sensitive to this parameter. It can be seen that, in the case of pure dust, the porosity lies in the range $52 \%$ to $72 \%$, in excellent agreement with our first analysis based on powdered rocks. The presence of ice causes the porosity range to decrease slightly, but it cannot be less than $\approx 50 \%$. This result is well in line with our present understanding of cometary nuclei as highly porous, low-density bodies as confirmed by the Deep Impact mission to comet 9P/Tempel 1. A'Hearn (2008) concludes that the porosity averaged over its entire nucleus is well above $50 \%$, reaching $75 \%$ in the surface layers on the basis of the luminous efficiency of the impact.

To summarize our findings and facilitate further discussion, we consider three typical solutions that sample the central axis of the allowed regions and use rounded numbers for simplicity. Solution A has no ice, a dust fraction of $35 \%$, and a porosity of $65 \%$. Although allowed by our constraint on the permittivity, this solution is probably unlikely since the thickness of the ice-free layer is expected to be much less than the penetration depth of the radar beam, as already discussed. Solution B has an ice fraction of $10 \%$, a dust fraction of $30 \%$, and a porosity of $60 \%$. Solution $\mathrm{C}$ has an ice fraction of $20 \%$, a dust fraction of
$23 \%$, and a porosity of $57 \%$. We note that the above fractions are volumetric.

We are aware that such high porosities may question the validity of the assumption of specular scattering inherent to the simple procedure of deriving the permittivity. In the review of Harmon et al. (2004), several nuclei have significant polarization ratios (their Fig. 5), which indeed argues against predominantly specular scattering. We note that this ratio for $67 \mathrm{P} / \mathrm{C}-\mathrm{G}$ is unknown and that there is presently no alternative to deriving the permittivity.

\section{Implications for the density of the nucleus}

If we assume that the nucleus subsurface layer probed by the radar beam is homogeneous, we can estimate its bulk density from our results coming from the ternary diagram, but we first have to specify the densities of the two phases, ice and dust. Hexagonal water ice has a bulk density of $920 \mathrm{~kg} \mathrm{~m}^{-3}$. The question is more complex for the dust phase, and we now discuss this question in detail with the additional purpose of justifying our choice of materials in our analysis (Sect. 5). To do so, we consider various interplanetary matters that can potentially be associated to comets and whose density can be measured or reliably inferred.

\subsection{Meteorites}

The association of meteorites to cometary material is far from being straightforward and is still a matter of debate. A first approach consists in considering primitive meteorites of petrologic type 3 since they have experienced none or very limited aqueous and thermal alterations since their formation. There are two potential candidates.

- The ordinary chondrites: they have an average grain density of $3540 \mathrm{~kg} \mathrm{~m}^{-3}$, very close to their bulk density of $3220 \mathrm{~kg} \mathrm{~m}^{-3}$ because of their very low porosity (Consolmagno et al. 2008).

- The CV carbonaceous chondrites: the two subgroups, oxidized and reduced, have slightly different average grain densities, 3300 and $3540 \mathrm{~kg} \mathrm{~m}^{-3}$, respectively, but different porosities, resulting in average bulk densities of respectively 2790 and $3120 \mathrm{~kg} \mathrm{~m}^{-3}$ (Consolmagno et al. 2008).

A second approach consists in considering the most chemically primitive meteorites based on the abundance of the nonvolatile elements similar to the Sun, that is the CI carbonaceous chondrites. The high porosity of CI's (up to 35\%) complicates the measurement of their densities, and different techniques yield different results. For the bulk and grain densities, Britt \& Consolmagno (2000) give 2120 and $2270 \mathrm{~kg} \mathrm{~m}^{-3}$, in that order, and Consolmagno et al. (2008) give 1600 and $2460 \mathrm{~kg} \mathrm{~m}^{-3}$. Specifically for the Orgueil CI1 chondrite, published values of the grain density are $2430 \mathrm{~kg} \mathrm{~m}^{-3}$ (Consolmagno \& Britt 1998) and $2760 \mathrm{~kg} \mathrm{~m}^{-3}$ (Bland et al. 2004), where the latter value being close to the density of $2870 \mathrm{~kg} \mathrm{~m}^{-3}$ for our mixture (Sect. 5).

\subsection{Meteoroids}

Modeling the penetration of meteors in the Earth atmosphere allows to infer their density and combining with trajectory analysis, to establish a connection with the parent bodies. In this framework, Kikwaya et al. (2011) studied 107 optical meteors (mm-sized particles) and found meteoroids with JFC-like orbits having densities clumping in the $2700-3500 \mathrm{~kg} \mathrm{~m}^{-3}$ with three 
Table 3. Density of the subsurface of the nucleus of comet $67 \mathrm{P} / \mathrm{C}-\mathrm{G}$ for three solutions of the ice and dust fractions, A, B, and C (see text for detail).

\begin{tabular}{lccc}
\hline \hline \multicolumn{1}{c}{ Solution \# } & $\mathrm{A}$ & $\mathrm{B}$ & $\mathrm{C}$ \\
\hline$f_{\text {ice }}$ & 0 & 10 & 20 \\
$f_{\text {dust }}$ & 35 & 30 & 23 \\
Porosity $(\%)$ & 65 & 60 & 57 \\
Density $\left(\rho_{\text {dust }}=2000\right)$ & 700 & 700 & 580 \\
Density $\left(\rho_{\text {dust }}=2500\right)$ & 900 & 840 & 680 \\
Density $\left(\rho_{\text {dust }}=3000\right)$ & 1050 & 1000 & 780 \\
Density $\left(\rho_{\text {dust }}=3500\right)$ & 1230 & 1050 & 880 \\
\hline
\end{tabular}

Notes. $f_{\text {ice }}, f_{\text {dust }}$ : volumetric fractions of ice and dust. $\rho_{\text {dust }}$ : density of the dust phase $\left(\mathrm{kg} \mathrm{m}^{-3}\right)$.

outliers at 925,1230 , and $4000 \mathrm{~kg} \mathrm{~m}^{-3}$ altogether averaging to $3100 \mathrm{~kg} \mathrm{~m}^{-3}$.

\subsection{Application to the nucleus of $67 P / C-G$}

The relevant densities to be used to estimate the bulk density of the subsurface of the nucleus of $67 \mathrm{P} / \mathrm{C}-\mathrm{G}$ are the grain densities since the porosity is inherently part of our modeling as illustrated by the ternary diagram. A range of grain density from 2000 to $3500 \mathrm{~kg} \mathrm{~m}^{-3}$ widely encompasses the different possibilities summarized above, and we accordingly consider four values, 2000, 2500, 3000, and $3500 \mathrm{~kg} \mathrm{~m}^{-3}$. The corresponding bulk densities for our three solutions $\mathrm{A}, \mathrm{B}$, and $\mathrm{C}$ sampling the region allowed by a dielectric permittivity of 2 are given in Table 3 , and they range from $\approx 600$ to $\approx 1000 \mathrm{~kg} \mathrm{~m}^{-3}$ with the exception of the extreme case of solution A (no ice) and a grain density of $3500 \mathrm{~kg} \mathrm{~m}^{-3}$, leading to a bulk density of $1230 \mathrm{~kg} \mathrm{~m}^{-3}$; however, solution A is unlikely, as pointed out in Sect. 5. The laboratory measurements of Heggy et al. (2012) on mixtures of ice and LL5 meteorite sample at a dust-to-ice mass ratio of 1 and bulk densities ranging from $\approx 900$ to $\approx 2000 \mathrm{~kg} \mathrm{~m}^{-3}$ show that a permittivity of 2 is obtained at a density of $\approx 950 \mathrm{~kg} \mathrm{~m}^{-3}$ (see their Fig. 5), consistent with our result. In addition, A'Hearn (2008) derived an upper limit of $1600 \mathrm{~kg} \mathrm{~m}^{-3}$ for the surface layers of the nucleus of 9P/Tempel 1.

Whether the above density range of $\approx 600$ to $\approx 1000 \mathrm{~kg} \mathrm{~m}^{-3}$ obtained for the top $\approx 2.5 \mathrm{~m}$ layer may be extended to the overall nucleus of $67 \mathrm{P} / \mathrm{C}-\mathrm{G}$ raises the question of its internal structure and its homogeneity, a matter still widely open and subject to intense debates and speculations. We assume that this is the case for the immediate purpose of comparing our result with those on other nuclei. In their review of the structure and density of cometary nuclei for the Comets II book, Weissman et al. (2004) find a range of 500 to $1200 \mathrm{~kg} \mathrm{~m}^{-3}$ - well in line with our result "with a perhaps best current value of $0.6 \mathrm{~g} \mathrm{~cm}^{-3}$ " $\left(600 \mathrm{~kg} \mathrm{~m}^{-3}\right)$. As summarized in Table V of Lamy et al. (2007), the range for each individual nucleus is always quite broad, not to mention conflicting results between various authors. The situation stems from the present impossibility of measuring the mass and volume of nuclei so one has to resort to indirect methods to infer their density. A widespread one consists in modeling the nongravitational force, but it involves many assumptions, and results are generally regarded as very uncertain. Weissman et al. (2004) summarize the history of the revisions of the density of the nucleus of 1P/Halley, starting from 100-200 $\mathrm{kg} \mathrm{m}^{-3}$ (Rickman et al. 1987) to finally $500-1200 \mathrm{~kg} \mathrm{~m}^{-3}$ (Skorov \& Rickman 1999). In the case of the nucleus of 19P/Borrelly, Farnham \& Cochran (2002) find a range of $290-830 \mathrm{~kg} \mathrm{~m}^{-3}$ whereas
Davidsson \& Gutiérrez (2004) find a barely non-overlapping range of $180-300 \mathrm{~kg} \mathrm{~m}^{-3}$.

In the case of $67 \mathrm{P} / \mathrm{C}-\mathrm{G}$, the only information on the nucleus density comes from the non-gravitational force modeling performed by Davidsson \& Gutiérrez (2005). We now examine their results in detail. They find a range of density of $100-370 \mathrm{~kg} \mathrm{~m}^{-3}$ if the nominal change of the orbital period of the comet is considered but only an upper limit of $500 \mathrm{~kg} \mathrm{~m}^{-3}$ if the uncertainty in the period change is considered. Alternatively, considering water production rates within an envelope around the measured data, they were unable to constrain the nucleus properties and then suggested an upper limit of the density of $600 \mathrm{~kg} \mathrm{~m}^{-3}$. In view of the inherent difficulties with this method, it is, as usual, difficult to obtain a reliable density or even a density range, and we are not surprised by the apparent discrepancy with our range of 600 to $1000 \mathrm{~kg} \mathrm{~m}^{-3}$. In the case of a homogeneous nucleus, the two results are at best marginally compatible and would converge to a density for the whole nucleus of $\approx 600 \mathrm{~kg} \mathrm{~m}^{-3}$, the extreme border value of the two ranges. Keeping in mind that our result strictly applies to the top $\approx 2.5 \mathrm{~m}$ thick layer, another alternative is an inhomogeneous nucleus with different densities for its bulk volume and for its top layer. However, such an investigation is beyond the scope of the present study.

\section{Conclusion}

Thanks to our knowledge of the nucleus of comet $67 \mathrm{P} / \mathrm{C}-\mathrm{G}$ based on recent optical observations, we have been able to reconsider the radar observations obtained in 1982 at the Arecibo observatory and derive valuable constraints on the subsurface structure and density of this nucleus. This shows the importance and the power of combining radar and optical data for the study of comet nuclei, even when these observations are spread out in time.

In the absence of a detectable radar echo, we determined a maximum radar cross section of $0.7 \mathrm{~km}^{2}$, leading to a maximum radar albedo of 0.05 . This low albedo probably results from a combination of low radar reflectivity material and a lightly packed upper layer of the nucleus with substantial roughness, consistent with its low thermal inertia. Based on radar observations of other cometary nuclei and asteroids, it is unlikely that the albedo can be lower than 0.04 so that we were able to constrain the dielectric permittivity of the subsurface layer to a narrow range of 1.9 to 2.1. Laboratory measurements and our modeling of mixtures of ice and dust led to a porosity in the range of approximately 55 to $65 \%$ and a density in the range of $\approx 600$ to $\approx 1000 \mathrm{~kg} \mathrm{~m}^{-3}$ for the top $\approx 2.5 \mathrm{~m}$ layer of the nucleus. If this range of density is representative of the whole nucleus, i.e., if it is fairly homogeneous, our range and the one obtained by Davidsson \& Gutiérrez (2005) from non-gravitational force modeling are at best marginally compatible illustrating our present ignorance of such a basic physical property and ultimately of the importance of the Rosetta mission, which will allow the very first direct and therefore unambiguous measurement. A second, equally frustrating point of ignorance concerns the internal structure of cometary nuclei, opening the possibility that their bulk density could be less than that of the subsurface layers. This again points to the importance of the Rosetta mission and particularly of the CONSERT radio experiment that will probe the interior of a nucleus for the first time.

Finally, we recall that the success of the landing of the PHILAE surface module remains critically dependent upon both the bulk density of the nucleus and of the mechanical properties of its surface layer. As far as the final approach is concerned, 
it would be safe with a bulk density close to $350 \mathrm{~kg} \mathrm{~m}^{-3}$, but a higher value as derived in this present study, if it applies to the whole nucleus, would clearly present some risks. Touchdown and anchoring on the surface, and later drilling will have to cope with a probable highly porous subsurface, although it would be alleviated by the weak gravity of the nucleus.

Acknowledgements. We are grateful to P. Beck and I. Buttarazzi (IPAG/UJF Grenoble) for their mineralogical and dielectric modeling of the Orgueil meteorite. We thank G. Pettengill and I. Shapiro for their participation in the 1982 radar observations, and G. Consolmagno and P. Vernazza for very helpful advice.

\section{References}

A’Hearn, M. F. 2008, Space Sci. Rev., 138, 237

Bland, P. A., Cressey, G., \& Menzies, O. N. 2004, Meteoritics, 39, 3

Boissier, J., Bockelée-Morvan, D., Biver, N., et al. 2014, Icarus, 228, 197

Britt, D. T., \& Consolmagno, G. J. 2000, Icarus, 146, 213

Campbell, M. J., \& Ulrichs, J. 1969, 74, J. Geophys. Res., B25, 5867

Consolmagno, G. J., \& Britt, D. T. 1998, Meteoritics, 33, 1231

Consolmagno, G., Britt, D., \& Macke, R. 2008, Chem. Erde, 68, 1

Davidsson, B. J. R., \& Gutiérrez, P. J. 2004, Icarus, 168, 392

Davidsson, B. J. R., \& Gutiérrez, P. J. 2005, Icarus, 176, 453

De Sanctis, M. C., Capria, M. T., \& Coradini, A. 2006, Icarus, 38, 1906

Farnham, T, L., \& Cochran, A. L. 2002, Icarus, 160, 398

Groussin, O., Lamy, P. L., \& Jorda, L. 2010, Planet. Space Sci., 58, 904

Gutiérrez, P. J., Jorda, L., Samarasinha, N. H., \& Lamy, P. 2005, Planet. Space Sci., 53, 1135

Hallikainen, M. T., Ulaby, F. T., \& Abdelrazik, M. 1986, IEEE Trans. Ant. Prop., AP-34, 1329

Harmon, J. K., \& Nolan, M. C. 2005, Icarus, 176, 175

Harmon, J. K., Campbell, D. B., Hine, A. A., Shapiro, I. L., \& Marsden, B. G. 1989, ApJ, 338, 1071

Harmon, J. K. Campbell, D. B, Ostro, S. J., \& Nolan, M. C. 1999, Planet. Space Sci., 47, 1409

Harmon, J. K., Nolan, M. C., Ostro, S. J., \& Campbell, D. B. 2004, in Comets II, eds. M. C. Festou, H. U. Keller, \& H. A. Weaver (Tucson: Univ. of Arizona Press), 265
Harmon, J. K., Nolan, M. C., Margot, J.-L., et al. 2006, Icarus, 184, 285

Harmon, J. K., Nolan, M. C., Giorgini, J. D., \& Howell, E. S. 2010, Icarus, 207, 499

Harmon, J. K., Nolan, M. C., Howell, E. S., Giorgini, J. D., \& Taylor, P. A. 2011, ApJ, 734, L2

Heggy, E, Palmer, E. M., Kofman, W., Clifford, S. M., et al. 2012, Icarus, 221 925

Hilchenbach, M., Rosenbauer, H., \& Chares, B. 2004, in The New ROSETTA Targets, eds. L., Colangeli, H. Rosenbauer, \& Chares (Dordrecht, Boston, London: Kluwer Acad. Pub.), 289

Jorda, L., Lamy, P., Faury, G., et al. 2007, Icarus, 187, 208

Kamoun, P. G. 1983, Radar Observations of Cometary Nuclei, Ph.D. Thesis, MIT

Kamoun, P. G., Pettengill, G. H., \& Shapiro, I. I. 1982a, in Comets, ed. L. L. Wilkening (Tucson: Univ. of Arizona Press), 288

Kamoun, P. G., Campbell, D. B., Ostro, S., Pettengill, G. H., \& Shapiro, I. I. 1982b, Science, 216, 293

Kamoun, P. G., Campbell, D., Pettengill, G. H., \& Shapiro, I. 1999, PSS, 47, 23

Kikwaya, J.-B., Campbell-Brown, M., \& Brown, P. G. 2011, A\&A, 530, A113

Küppers, M., Bertini, I., Fornasier, S., et al. 2005, Nature, 437, 987

Lamy, P. L., Toth, I., Weaver, H. A., et al. 2006, A\&A, 458, 669

Lamy, P. L., Toth, I., Davidsson, B. J. R., et al. 2007, Space Sci. Rev., 128, 23

Lamy, P. L., Toth, I., Groussin, O., et al. 2008, A\&A, 489, 777

Lisse, C. M., VanCleve, J., Adams, A. C., et al. 2006, Science, 313, 635

Lowry, S. C., Fitzsimmons, A., Jorda, L., et al. 2006, BAAS, 38, 492

Lowry, S. C., Duddy, S. R., Rozitis, B., et al. 2012, A\&A, 548, A12

Magri, C., Nolan, M. C., Ostro, S. J., \& Giorgini, J. D. 2007, Icarus, 186, 126

Matzler, C. 1996, IEEE Trans. Geosci. Remote Sens., 34, 573

Mitchell, D. L., Ostro, S. J., Rosema, K. D., et al. 1995, Icarus, 118, 105

Rickman, H. Kamel, L., Festou, M. C., \& Froeschle, Cl. 1987, ESA, Proc. of the International Symp. on the Diversity and Similarity of Comets, 278, 471

Rosenberg, E. D., \& Prialnik, D. 2009, Icarus, 201, 740

Sagdeev, R. Z., Elyasberg, P. E., \& Moroz, V. I. 1988, Nature, 331, 241

Shepard, M. K., Clark, B. E., Ockert-Bell, M, et al. 2010, Icarus, 208, 221

Sihvola, A. 2004, Electromagnetic mixing formulas and applications. The Institution of Electrical Engineers (London: United Kingdom), 242

Skorov, Yu. V., \& Rickman, H. 1999, Planet. Space Sci., 47, 935

Sykes, M. V., \& Walker, R. G. 1992, Icarus, 95, 180

Weissman, P. R., Asphaug, E., \& Lowry, S. C. 2004, in Comets II, eds. M. C. Festou, H. U. Keller, \& H. A. Weaver (Tucson: Univ. of Arizona Press), 337 